\title{
Correlated atomic motions in glassy selenium
}

\author{
F. J. Bermejo and M. García-Hernández \\ Instituto de Estructura de la Materia, Consejo Superior de Investigaciones Cientifícas, Serrano 123, E-28006 Madrid, Spain \\ T. Mason \\ Department of Solid State Physics, Risфe National Laboratory, DK-2000 Roskilde, Denmark \\ J. L. Martínez \\ Instituto de Ciencia de Materiales, Consejo Superior de Investigaciones Científicas, Department Física de Materiales C-IV, \\ Facultad de Ciencias, Universidad Autónoma de Madrid E-28049, Spain \\ E. Enciso \\ Instituto de Estructura de la Materia, Consejo Superior de Investigaciones Científicas, Serrano 123, E-28006 Madrid, Spain
}

A. Criado

Departamento de Física de la Materia Condensada, Universidad de Sevilla and Instituto de Ciencia de Materiales, P.O. Box 1065, E-41080 Sevilla, Spain

(Received 1 March 1994; revised manuscript received 22 June 1994)

\begin{abstract}
The elastic and total (energy-integrated) structure factors for glassy selenium have been measured by means of neutron spectroscopy within the temperature range $15 \mathrm{~K} \leq T \leq 310 \mathrm{~K}$. The correlated nature of the atomic vibrations gives rise to marked features in the total (energy-integrated) inelastic structure factors defined as the difference between the total and elastic intensities. Also, both structure factors show rather distinct dependences with temperature, which are discussed in some detail.
\end{abstract}

\section{INTRODUCTION}

The microscopic, space-dependent motions of a substantial number of glassy materials have been investigated by means of experimental probes such as neutron scattering (for a relatively recent appraisal see Ref. 1), alongside with theoretical or computer simulation approaches. ${ }^{2}$ However, and leaving aside the pioneering work performed on monoatomic (metallic binary) glasses $^{3}$ or those regarding archetypal glasses formed by well-defined structural units, such as amorphous silica and oxide glasses (see, for instance, Ref. 4), most of the reported studies have focused on the study of relatively high-energy excitations (bond stretching and bendings ${ }^{5}$ ), aiming to contrast the results of theoretical calculations cast in terms of the normal modes of small atomic clusters. ${ }^{6}$

The purpose of the present work is to present recent results regarding vitreous $\mathrm{Se}$, one of the most widely studied topologically disordered glasses, using for the purpose measurements of the elastic (zero-energy transfer) and total structure factor, carried out under conditions that make amenable a detailed study (with high statistical accuracy) of the neutron-scattering cross sections (integrated in energy) of those excitations responsible for the well-documented anomalies regarding thermal (the $C / T^{3}$ bump in the specific heat at $T=6 \mathrm{~K}$ ) and transport (the plateau in the heat conductivity) properties. Contrary to previous studies, ${ }^{7}$ where the interest was centered on the detailed study of the generalized (density of states) and wave-vector-dependent (dynamic structure factors) frequency spectra, our aim here is to investigate the $Q$ dependence of magnitudes, which can be accessed from experimental means with relative ease, thus enabling a detailed study of the wave-vector and temperature dependences of the spectra. Although detailed information regarding the time-dependent atomic dynamics cannot be derived from the kind of measurements presented here, they provide valuable information regarding the dependence with both momentum transfers and temperature of the ratios of the elastic to the total intensity as well as of the total inelastic intensity. As will be shown below, the analysis of such quantities in terms of microscopic dynamical models will serve to infer the highly correlated nature of the atomic dynamics at length scales several times larger than that characteristic of the self-atomic (single-particle) motion.

\section{EXPERIMENTAL AND CALCULATION DETAILS}

\section{A. Experiments}

The measurements were carried out using the TAS7 cold triple-axis instrument located at one of the cold (curved) neutron guides of the DR3 reactor at the Risoe National Laboratory, Roskilde (Denmark), which enables us to perform measurements free of contamination from higher harmonics, as attested from the spectra of the empty cell. An incident neutron energy $E_{0}=1.956 \mathrm{THz}$ was selected, and the spectrometer was set up using col- 
limations of $30^{\prime}, 20^{\prime}, 20^{\prime}, 40^{\prime}$, which resulted in an energy resolution at the elastic line of about $0.05 \mathrm{THz}$ [full width at half maximum (FWHM)] and a resolution in momentum transfers of $Q \approx 0.04 \AA^{-1}$. The chosen experimental conditions imply that for moderately low temperatures only excitations below $E_{0}$ (i.e., covering the first broad peak in the vibrational frequency distribution ${ }^{7}$ ) are being sampled. Two sets of measurements were carried out within the temperature range $15 \mathrm{~K} \leq T \leq 310 \mathrm{~K}$ $\left(T_{g} \approx 313 \mathrm{~K}\right.$ for this long annealed sample) comprising most of the glass stability range. Measurements of the elastic cross section for each temperature were followed by the measurements of the total elastic cross section, using the same spectrometer setup. Under these experimental conditions, the usable range of momentum transfers was $0.4 \AA^{-1} \leq Q \leq 2.6 \AA^{-1}$, and to ensure the same statistical accuracy in both sets of measurements, every data point was counted up to 10000 monitor counts. The sample consisted in a machined niobium slab cell of dimensions $5 \mathrm{~cm}$ high, $6 \mathrm{~cm}$ wide, and an internal thickness of $0.3 \mathrm{~cm}$ filled with selenium powder which was heated up to $640 \mathrm{~K}$ for $2 \mathrm{~h}$ and subsequently quenched into a mineral oil bath. Diffraction patterns of the same sample carried out previously using a larger extent in reciprocal space $^{7}$ did not reveal the presence of any Bragg reflection. Once quenched, the sample was shown to be stable at room temperature for at least six months, and the present measurements correspond to such an annealed sample. Special care was put into an accurate evaluation of the absorption corrections, which become particularly severe for this relatively highly absorbing material. Our own computer code was employed for such a purpose ${ }^{8}$ and the contribution of multiply scattered neutrons was evaluated using the DISCUs code, using as a scattering kernel spectra available from the computer simulations already reported. The contribution of multiple scattering to the elastic line as well as to the total elastic cross section (evaluated from integration of this contribution over the kinematically accessible transfers) never exceeded $\approx 4 \%$ and were subtracted from the total intensities by means of the factor method. In order to account for the resolution broadening of the strictly elastic peak, calculations performed using the RESCAL $\operatorname{code}^{9}$ were compared with that measured using a vanadium standard, thus enabling us to adjust the values of some of the instrumental parameters (beam divergences). As a result, resolution functions slowly varying with $Q$ (maximum percentage variation of about $7 \%$ ) were calculated, and the height of the measured elastic intensity corrected accordingly. The measured cross sections were then converted into structure factors and the normalization was carried out by comparison with the structure factors derived from simulational means. ${ }^{7}$ A sample of a set of representative structure factors is shown in Fig. 1. No detailed comparison with previously published spectra is possible due to the far different experimental conditions.

\section{B. Structure factors \\ and their dependence with temperature}

In what follows we shall consider the glassy Se sample as a macroscopically large molecule composed of $\mathrm{N}$ atoms (although, as will be shown below the characteristic coherence lengths bound the dimensions that matter down to a few tens of $\AA$ ), interacting through harmonic potentials, and therefore closed from expressions for the terms entering the phonon expansion of the $S(Q, \omega)$ dynamic structure factor, are available. ${ }^{10-12}$ The elastic (zero-phonon) term becomes

$$
\begin{aligned}
S^{(0)}(Q, \omega) & =\delta(\omega) \frac{1}{N} \sum_{l, m} I_{l, m}^{(0)}(Q), \\
I_{l, m}^{(0)}(Q) & \approx\left\langle e^{i \mathbf{Q} \cdot \mathbf{r}_{l}, m}\right\rangle_{\Omega}\left\langle^{-\left(W_{l}(\mathbf{Q})+W_{m}(\mathbf{Q})\right)}\right\rangle_{\Omega} \\
& =j_{0}\left(Q r_{l m}\right)\left\langle e^{-2 W(\mathbf{Q})}\right\rangle_{\Omega},
\end{aligned}
$$

where the sum runs over all atoms of the system composed by $N$ scattering units, $\mathbf{r}_{l, m}$ stands for the equilibrium distance between atoms $l, m, j_{n}(x)$ denote spherical Bessel functions, and the subindex $\Omega$ stands for the orientational averaging. The exponent in the last expression is given by

$$
2 W(Q)=\frac{3 \hbar}{2 M_{\mathrm{Se}}} \int_{0}^{\omega_{\max }} d \omega \frac{Z(\omega)}{\omega}\left\langle(\mathbf{Q} \cdot \gamma)^{2}\right\rangle_{\omega}[2 n(\omega)+1]
$$

where $\omega_{\max }$ represents the maximum frequency of $Z(\omega)$, the generalized vibrational density of states, $M_{\mathrm{Se}}$ is the atomic mass of a selenium atom, $\gamma$ stands for the normalized vector amplitudes of modes for a given fequency $\omega$, over which an average is taken as specified by the angular brackets, and $2 n(\omega)+1$ is a Bose factor. ${ }^{13} \mathrm{~A}$ more compact definition of the elastic term can be written upon the introduction of a rigid structure factor as ${ }^{12,14}$

$$
\begin{aligned}
S^{(0)}(Q, \omega) & =\delta(\omega) S_{R}(Q) e^{-2 W(Q)} \\
S_{R}(Q) & =\frac{1}{N} \sum_{l, m} j_{0}\left(Q r_{l m}\right)
\end{aligned}
$$

The static structure factor is given in terms of the phonon expansion as

$$
\begin{aligned}
S(Q) & =S^{(0)}(Q, \omega)+S^{(1)}(Q)+S^{(2)}(Q)+\cdots \\
& =\int_{-E_{\min }}^{E_{\max }} d \omega \cosh (\hbar \omega \beta / 2) S(Q, \omega) \\
& \equiv \frac{1}{N} \sum_{l, m}\left\langle e^{i \mathbf{Q} \cdot \mathbf{r}_{l, m}} e^{-2 W_{l, m}^{\prime}(\mathbf{Q})}\right\rangle_{\Omega}
\end{aligned}
$$

where the integration limits are set by the neutron kinematics and, due to coherent interference effects, ${ }^{11,15}$ the exponential term, which accounts for thermal disorder entering this structure factor, is now

$$
2 W_{l, m}^{\prime}(\mathbf{Q})=\frac{\hbar}{4 N M_{\mathrm{Se}}} \sum_{\lambda}\left(\mathbf{Q} \cdot\left\{\gamma_{l}(\lambda)-\gamma_{m}(\lambda)\right\}\right)^{2} \frac{\left(2 n_{\lambda}+1\right)}{\omega^{\lambda}}
$$


where the $\gamma_{l}-\gamma_{m}$ terms give account of the correlation between the amplitudes of a normal vibration of frequency $\omega^{\lambda}$ taken over all atoms separated by a distance $\mathbf{r}_{l, m}$. Such an exponential, usually referred to as the Debye-Waller term in the glass transition literature usually shows a substantial structure in $Q$ with well-defined phase relationships with the structure factor. ${ }^{16}$ Its factorization outside the orientational average can only be carried out for long times, where it becomes identical to $2 W(Q)$, and such an exercise is precluded in the present work, since our purpose is the study of properties corresponding to intermediate times.

The relevant $S^{n}(Q) n$-phonon terms of Eq. (5) have been evaluated by Carpenter and Pelizzari [see Eqs. (19)-(28) of Ref. 11] and the inelastic structure factors contributing to the total intensity can be readily calcu- lated after integration over energy transfers as

$$
\begin{aligned}
& S^{(1)}(Q)=\sum_{l, m} \sum_{\lambda} \alpha_{\lambda}^{(1)} F_{l, m}^{(1, \lambda)}(Q), \\
& S^{(2)}(Q)=\sum_{l, m} \sum_{\lambda} \alpha_{\lambda}^{(2)} F_{l, m}^{(2, \lambda)}(Q)+\alpha_{\lambda, \lambda^{\prime}}^{(2)} F_{l, m}^{\left(2, \lambda, \lambda^{\prime}\right)}(Q),
\end{aligned}
$$

where the sums run over different $l, m$ atom pairings and normal modes labeled by $\lambda$, and the population factors $\alpha$ and the $F_{l, m}(\lambda)$ inelastic structure factors for normal modes with frequencies $\omega^{\lambda}$ are the same than those given in Ref. 11.

From the experimental side, the absence of sharp Bragg reflections precludes the direct measurement of either $2 W(Q)$ or $2 W^{\prime}(\mathbf{Q})$ from experiments measuring only one of the structure factors, although the former can be

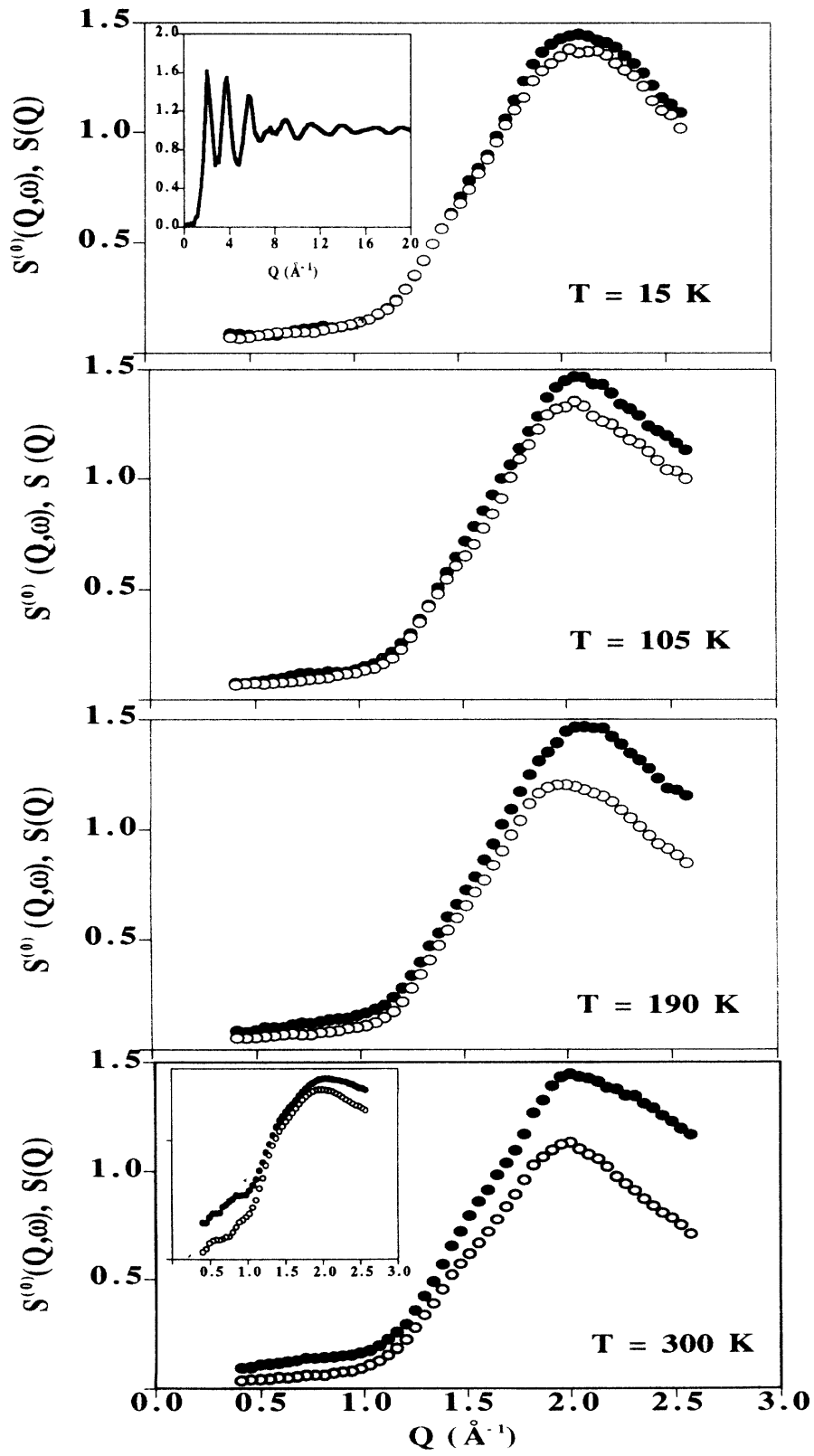

FIG. 1. $S^{(0)}(Q, \omega)$ (open symbols) and $S(Q)$ (filled symbols) for some temperatures comprising the explored range (given in the inset). The inset in the uppermost figure represents the $S(Q)$ structure factor at $T=15 \mathrm{~K}$ calculated from MD simulation (Ref. 4) and shown for comparison purposes. The inset in the lowest graph shows both structure factors in a logarithmic scale, evidencing the presence of a small shoulder at $Q \approx 0.8 \AA^{-1}$ more clearly visible in the strictly elastic function. The error bars due to counting statistics are far smaller than the symbols. 
measured if incoherent scattering samples were available (no suitable isotope for $\mathrm{Se}$ ). A common practice in recent times ${ }^{17}$ has lead to estimating an averaged (over all atoms) value of the Debye-Waller factor from the ratio of the strictly elastic to the total structure factors. Within the approach followed here, such a ratio results in

$$
\begin{aligned}
f(Q) & =\frac{S^{(0)}(Q, \omega)}{S(Q)} \\
& =\frac{S^{(0)}(Q, \omega)}{S^{(0)}(Q, \omega)+S^{(1)}(Q)+S^{(2)}(Q) \cdots}
\end{aligned}
$$

a quantity comprising relevant information regarding the correlation of atomic motions, although its interpretation becomes extremely involved.

The total (frequency-integrated) inelastic intensity, which is also experimentally accessible, finally provides a direct access to the inelastic structure factors, since

$$
\begin{aligned}
I_{\text {inel }}(Q) & =S(Q)-S^{(0)}(Q, \omega) \\
& =S^{(1)}(Q)+S^{(2)}(Q)+\cdots
\end{aligned}
$$

The measurement of the energy-integrated inelastic intensity thus constitutes an excellent test to investigate the wave-vector dependence of the correlation effects, specially if workable models to perform the averages on the $F(Q)$ structure factors are brought forward.

To investigate the temperature dependence of both structure factors and gain some physical insight, it is convenient, for calculation purposes, to replace the exponential in Eq. (3) by $1-\left[W_{0}(Q)+W_{T}(Q)\right]$ comprising zeropoint motion and temperature-dependent effects. The isobaric derivative with respect to the temperature of the elastic term becomes

$$
\frac{\partial S^{(0)}(Q, \omega)}{\partial T}=\frac{\partial S_{R}(Q)}{\partial T}[1-W(Q)]-S_{R}(Q) \frac{\partial W_{T}(Q)}{\partial T},
$$

where the first term on the right-hand side mainly contains contributions from thermal expansion effects and its value is some orders of magnitude smaller than the term containing the derivative of $W_{T}(Q)$. Since the derivative,

$$
\left.\frac{\partial W_{T}(Q)}{\partial T}=\frac{3 \hbar}{2 M_{\mathrm{Se}}} \int_{0}^{\omega_{\max }} d \omega \frac{Z(\omega)}{\omega}\left\langle(\mathbf{Q} \cdot \gamma)^{2}\right)\right\rangle_{\omega} \frac{\hbar \omega k_{B} \beta^{2} / 2}{\sinh ^{2}(\hbar \omega \beta / 2)}
$$

is a positive quantity for frequencies within the range of the integral, $S^{(0)}(Q, \omega)$ is expected to show a decrease with temperature with a dependence with wave vector governed by the product of $S_{R}(Q)$ and the wave-vector dependence of the derivative given by Eq. (12). If the sample were to be considered as microscopically isotropic (i.e., the atomic vibration amplitudes in all directions were the same), then the last term in Eq. (11) would be $\propto-S_{R}(Q) Q^{2}$, and therefore the derivative should exhibit extrema at $Q$ values where $S_{R}(Q)$ has maxima. Deviations from such simple behavior are thus expected if the isotropy condition is not fulfilled at microscopic scales as well as to the presence of higher-order (anharmonic) contributions to the total structure factor. A rather more complicated treatment follows regarding the $S(Q)$ structure factor. Here the temperature derivative will contain a sum of the contributions from the $n$-phonon expansion, with a first term given by Eq. (11), and the temperature dependence of the one-phonon contribution reads,

$\frac{\partial S^{(1)}(Q)}{\partial T}=\sum_{l, m} \sum_{\lambda} \frac{\partial \alpha_{\lambda}^{(1)}}{\partial T} F_{l, m}^{(1, \lambda)}(Q)+\alpha_{\lambda}^{(1)} \frac{\partial F_{l, m}^{(1, \lambda)}(Q)}{\partial T}$.

The contributions from the first terms in the summation can be readily evaluated as

$$
\begin{aligned}
\frac{\partial \alpha_{\lambda}^{(1)}}{\partial T} F_{l, m}^{(1, \lambda)}(Q)= & \frac{\hbar^{2}}{4 k_{B} T^{2}} \operatorname{csch}\left(\frac{\hbar \omega^{\lambda}}{2 k_{B} T}\right) \\
& \times \operatorname{coth}\left(\frac{\hbar \omega^{\lambda}}{2 k_{B} T}\right) F_{l, m}^{(1, \lambda)}(Q) .
\end{aligned}
$$

Such functions show a steep rise with temperatures up to $\hbar \omega^{\lambda} / k_{B}$ and become the dominant contribution to the increase of the inelastic intensity with temperature (in a harmonic approximation). Evaluation of the temperature dependence of the second terms obviously represents a difficulty, since no closed form expression is available for the structure factors thus requiring further approximations as will be shown below. In summary, the temperature dependence of $S(Q)$ contains a compensating effect arising from the negative derivative of the first (elastic) term and the positive ones from the inelastic intensities. Moreover, such dependence is also expected to exhibit a remarkable dependence with wave vector as it is governed by both, the interplay of the alluded opposing effects and from the $Q$-dependent terms entering the derivatives given by Eq. (13).

\section{RESULTS AND DISCUSSION}

The structure factors analyzed in this contribution correspond to the first most intense peak of the diffraction pattern of glassy Se. A closer look to the graphs displayed in Fig. 1, also reveals the presence of small humps at $Q \approx 0.8$ and $1.6 \AA^{-1}$, with a relative intensity depending upon the temperature. For wave vectors between $1 \AA^{-1} \leq Q \leq 2.4 \AA^{-1}$, the peak shapes can be roughly described by a Gaussian, and the temperature dependence of its parameters shows that (a) the peak maxima of both structure factors shift to lower $Q$ values from $2.13 \AA^{-1}$ at $T=15 \mathrm{~K}$ to $1.99 \AA^{-1}$ at $T=300 \mathrm{~K}$ for $S^{(0)}(Q, \omega)$, with a concomitant shift from $2.12 \AA^{-1}$ at $T=15 \mathrm{~K}$ to $2.08 \AA^{-1}$ at $T=300 \mathrm{~K}$ for 
$S(Q)$, and (b) a strong decrease with temperature of the intensity of $S^{(0)}(Q, \omega)$ is noticed, although such a decay $(\approx 22 \%)$, is not monotonous, since for temperatures below $60 \mathrm{~K}$ a plateau is observed. On the other hand the intensity of the peak in $S(Q)$ stays roughly

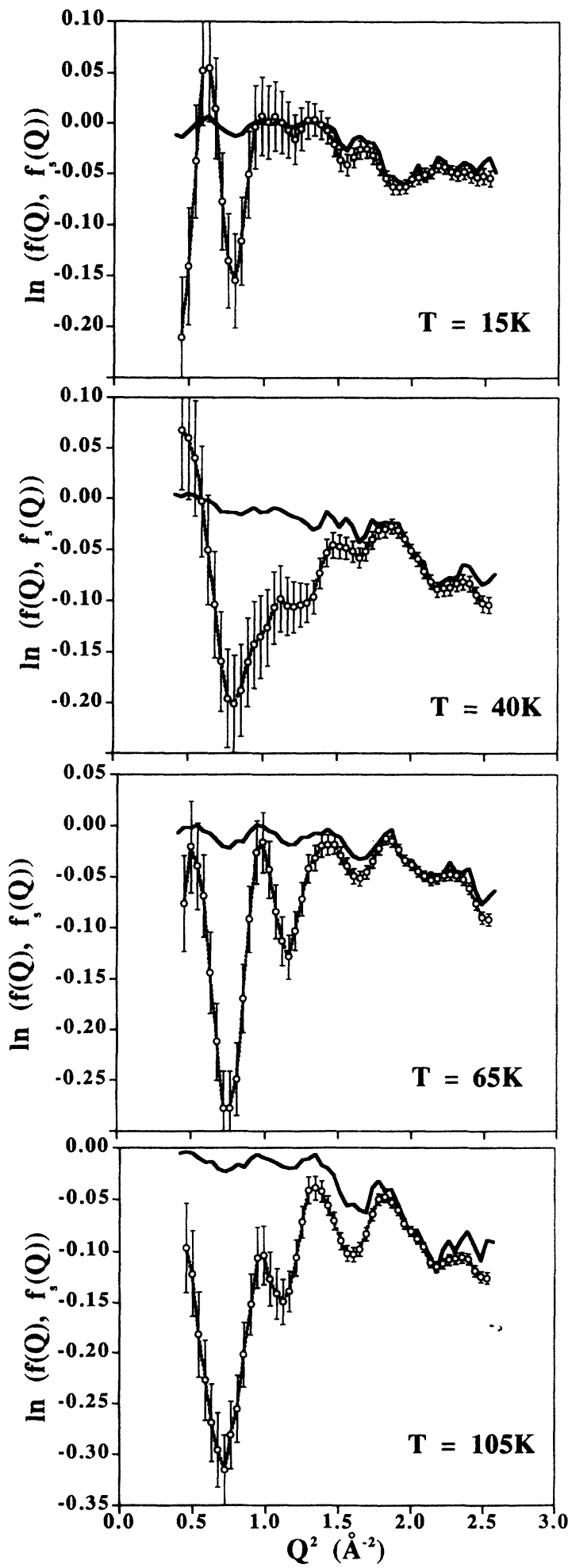

constant up to $190 \mathrm{~K}$ from where a decrease of a mere $3 \%$ is found. The temperature dependence of both structure factors can be compared with data regarding the first sharp diffraction peak in vitreous silica, ${ }^{18}$ where a small shift $\left(0.01 \AA^{-1}\right)$ has been found concomitantly with

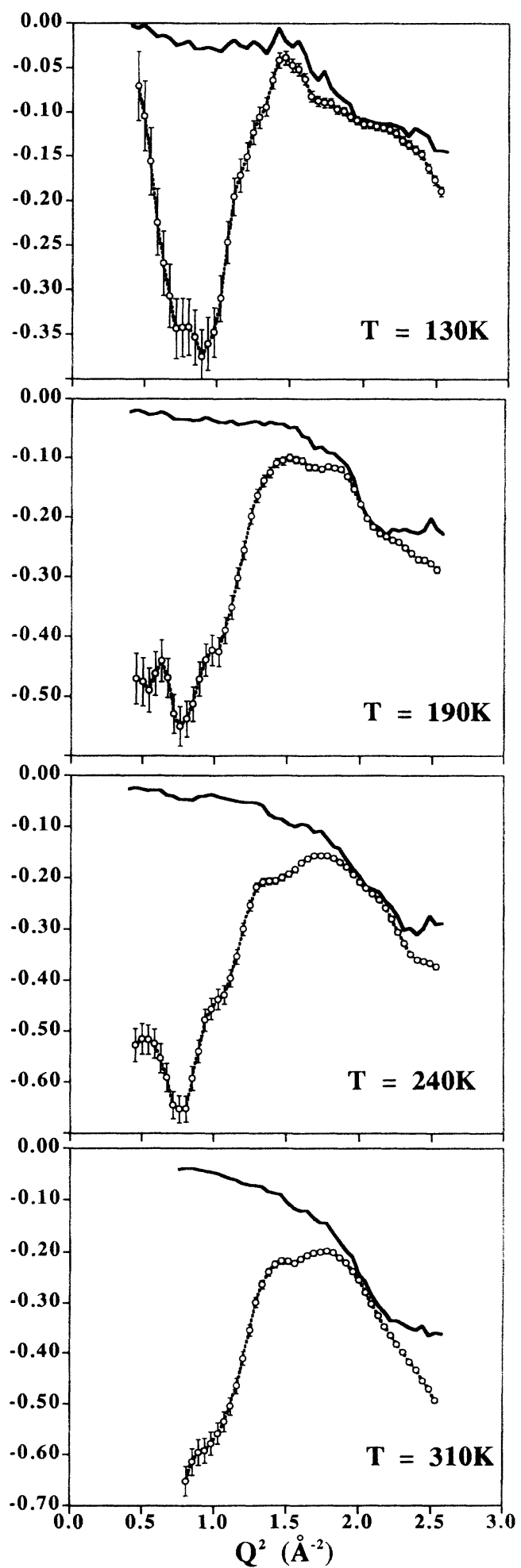

FIG. 2. A comparison of the ratios $f(Q)$ (open symbols with error bars) and $f_{s}(Q)$ (solid lines) for all the studied temperatures shown as a semilogarithmic plot vs $Q^{2}$. 
a large decrease in intensity. Also, the shift coefficient at constant pressure defined as $\alpha=-\left(1 / Q_{0}\right)\left(\partial Q_{0} / \partial T\right)$ where $Q_{0}$ stands for the wave vector corresponding to the peak maxima of both structure factors, results in values, for temperatures where the variation is almost linear [above $50 \mathrm{~K}$ in $S^{(0)}(Q, \omega)$ and above $100 \mathrm{~K}$ in $S(Q)$ ] of $1.27 \times 10^{-4} \mathrm{~K}^{-1}$ and $3.15 \times 10^{-5} \mathrm{~K}^{-1}$, respectively. Such value for the $S(Q)$ is of the same order of nugnitude of covalent glasses, ${ }^{19}$ whereas the one regarding the elastic component becomes several times larger. It is interesting to notice that the experimental values for the linear expansion coefficient ${ }^{20}$ show a strong rise up to $50 \mathrm{~K}$ and a subsequent smooth increase from $2.7 \times 10^{-5}$ at $50 \mathrm{~K}$ to $5.3 \times 10^{-5}$ at $300 \mathrm{~K}$ [i.e., the values of the volume expansivity are of the same order of magnitude than the coefficient for $S^{(0)}(Q, \omega)$, and therefore, as expected, such effects are mimicked by the elastic structure factor far more closer than $S(Q)$.

In what follows, and in order to describe more accurately the present findings it seems convenient to divide the results into those regarding the wave-vector dependence of the intensities at fixed temperatures and the dependences with temperature at fixed wave vectors.

As pointed out in the preceding section, the experimental quantities to be analyzed are $f(Q)$, the ratio of elastic to total (energy-integrated) intensities, the difference $S(Q)-S^{(0)}(Q)$, as well as some related magnitudes. In particular, the quantity $f_{s}(Q)=S^{(0)}(Q, \omega)-S(Q)+1$ has been proposed in some other studies on glassy dynamics as an empirical test regarding the correlations between vibrational motions of different atoms, ${ }^{10}$ since in the case of no correlation such quantity should approach the Lamb-Mössbauer factor. A set of plots of the functions $\ln \left[S^{(0)}(Q, \omega) / S(Q)\right]$ and $\ln \left[S^{(0)}(Q, \omega)-S(Q)+1\right]$ are shown in Fig. 2 in a semilogarithmic scale. Several features are worth remarking regarding these plots; first and foremost, the function $\ln \left[S^{(0)}(Q, \omega)-S(Q)+1\right]$ deviates significantly from the linear behavior characteristic of the self- (incoherent) atomic motion, since significant oscillations are present even at the highest explored temperatures. Also, the appearance of marked oscillations in $f(Q)$ for temperatures below $130 \mathrm{~K}$ without a clear phase relationship to the structure factors indicates that the atomic motions being sampled at these relatively low temperatures are additional to the plane wave excitations characteristic of sound waves. The fact that such oscillations get progressively blurred out as the temperature is raised can be rationalized if the multiphonon contributions are taken into account as it will be shown below. The deviation from linear behavior in $\ln \left[S^{(0)}(Q, \omega)-S(Q)+1\right]$, can thus be interpreted as arising from terms $\left\langle\left[\mathbf{Q} \cdot \gamma_{l}(\lambda)\right]^{2}\right\rangle_{\omega}$ in Eq. (2), which, due to correlations between the atomic motions do not average out to $\frac{1}{3} Q^{2} u^{2}$ in terms of a single average mean-square displacement. The behavior of $f(Q)$ at $Q$ values below $1.5 \AA^{-1}$ evidences the presence of noticeable structure. Similar findings have been reported from numerical simulations of Lennard-Jones systems close to the glass transition by Bengtzelius ${ }^{17}$ and from neutron experiments as recently discussed by us. ${ }^{16}$

Figure 3 shows the $I_{\text {inel }}(Q)$ inelastic intensities for some selected temperatures. As can be seen, additional oscillations to those expected on the basis of plane-wave excitations [i.e., expected to follow a $\propto Q^{2} S^{(0)}(Q, \omega)$ dependence] are seen at all temperatures, and again, the oscillating patterns get smeared out as the temperature is raised above $100 \mathrm{~K}$. A comparison of these quantities with the one derived from an harmonic lattice dynamics calculation for the trigonal polycrystal ${ }^{7}$ shown in the inset (i.e., frequency integrals over the calculated dynamic structure factor excluding the elastic component), reveals that the only features visible in the harmonic crystal within the same $Q$ range, are a small peak at $Q \approx 1.7$ $\AA^{-1}$, which originates from the first Bragg reflection, and the main peak at $Q \approx 2.1 \AA^{-1}$. A glance to the curves shown in Fig. 3, thus indicates that the additional intensity appearing below $Q \approx 1.5 \AA^{-1}$ should be considered as fingerprints of the glassy dynamics.

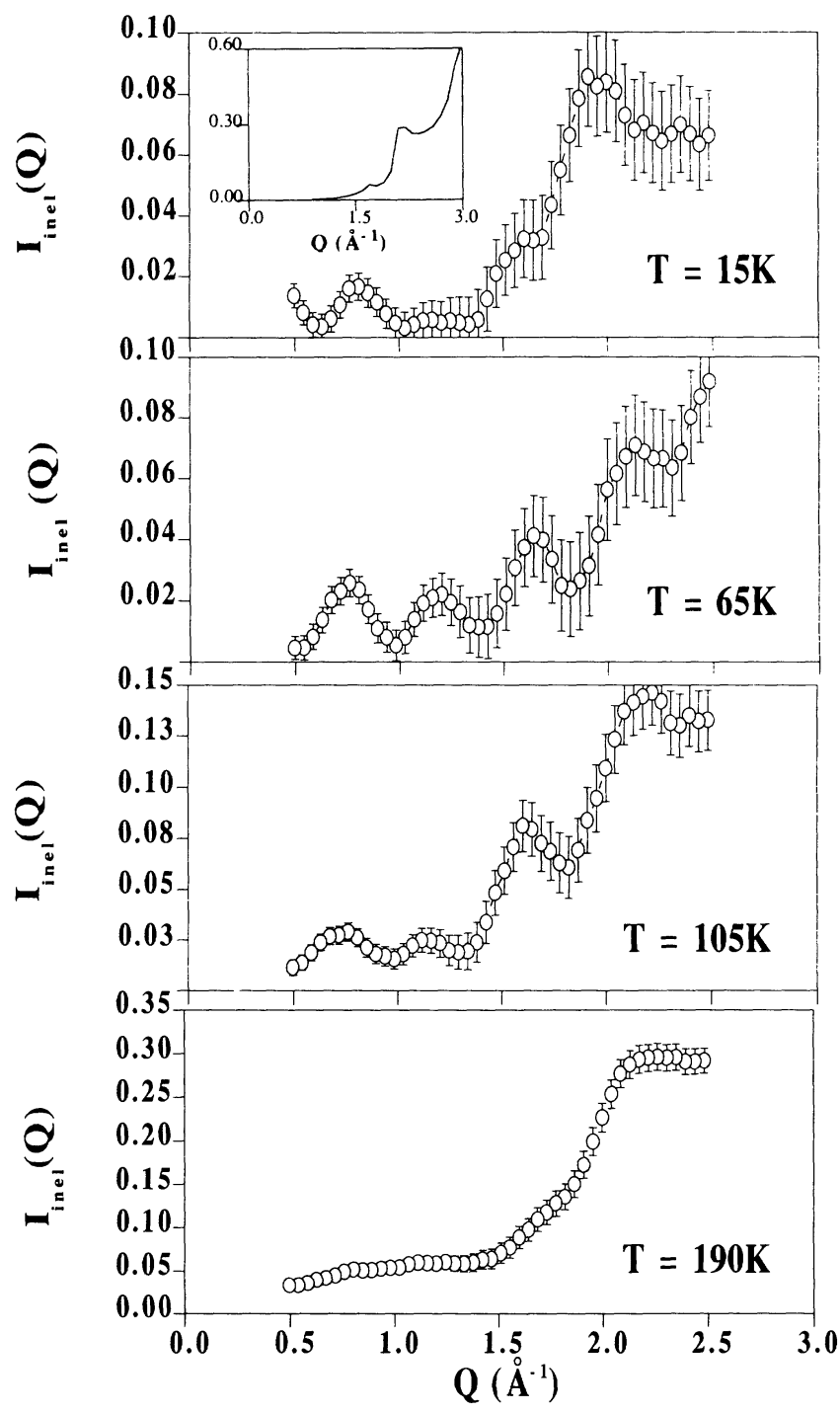

FIG. 3. Wave-vector dependence of the inelastic intensities for the temperature values given in the insets. The uppermost graph depicts this quantity as calculated for the trigonal polycrystal (see text). 
To get some insight into the origin of the observed patterns, consideration was made of the model proposed by Jäckle and Froböse, ${ }^{12}$ which basically extends that proposed by Carpenter and Pelizarri,,${ }^{11}$ and makes explicit use of a correlation function defined as the product of vector displacements $\boldsymbol{\gamma}_{l}^{\alpha} \cdot \boldsymbol{\gamma}_{m}^{* \boldsymbol{\beta}}$ averaged over all pairs of atoms separated by a distance $\mathbf{R}$ and over all modes of a given frequency. Within such a scheme, the inelastic intensity can be written as a convolution of the alluded correlation function and the elastic component as

$$
\begin{aligned}
I_{\text {inel }}(Q)= & \frac{\pi \hbar}{M_{\mathrm{Se}}} \int_{-E_{\min }}^{E_{\max }} d \hbar \omega \frac{Z(\omega)}{\omega}[n(\omega)+1] \\
& \times \int d^{3} \mathbf{q} S^{(0)}(|\mathbf{Q}-\mathbf{q}|, \omega) q_{\alpha} q_{\beta} C_{\alpha \beta}(\mathbf{q}, \omega)
\end{aligned}
$$

which enables the calculation of $I_{\text {inel }}(Q)$ if information regarding the static structure factor, the vibrational density of states as well as a prescription for the mentioned correlation function are available. ${ }^{21}$ For such a purpose, we have made use of the $Z(\omega)$, structure factor and $C_{\alpha \beta}(\mathbf{q}, \omega)$ as calculated from molecular dynamics (MD) simulations, ${ }^{7}$ and a comparison between experimental and calculated quantities is given in Fig. 4. As can be seen from Fig. 4(a), which regards low-temperature data enabling the use the one-phonon approximation on a safe ground, a poor agreement is found, even if the mode wave vectors $q_{\alpha, \beta}$ are calculated from the average dispersion frequencies $\omega_{0}(Q)$ and $\omega_{l}(Q)$ (i.e., the first normalized even frequency moments of the scattering law, see Ref. 7 for additional details), and their associated polarizations (phonon strengths) derived from previous work are used as input to model the correlation function. On the other hand, some additional structure seems to be related to excitations of a single selenium chain as shown in curve (c) of the figure, where the excitation frequency and polarization factors were taken from a previous calculation. ${ }^{7}$ However, the higher-frequency oscillation appearing in the experimental pattern cannot be reproduced using as input the above referred excitations. Since the lowestlying excitations of our system seem to be related to torsional librations about the chain bonds (the associated libration frequency is of about $1.5 \mathrm{THz}$ ), we have modeled the inelastic form factor of a four-atom segment using the geometric and force-constant data given in a previous communication, ${ }^{22}$ and following the same approach employed for the approximation of the inelastic structure factors. ${ }^{11}$ Assuming that, due to the relatively low temperature the torsional motion can be decoupled

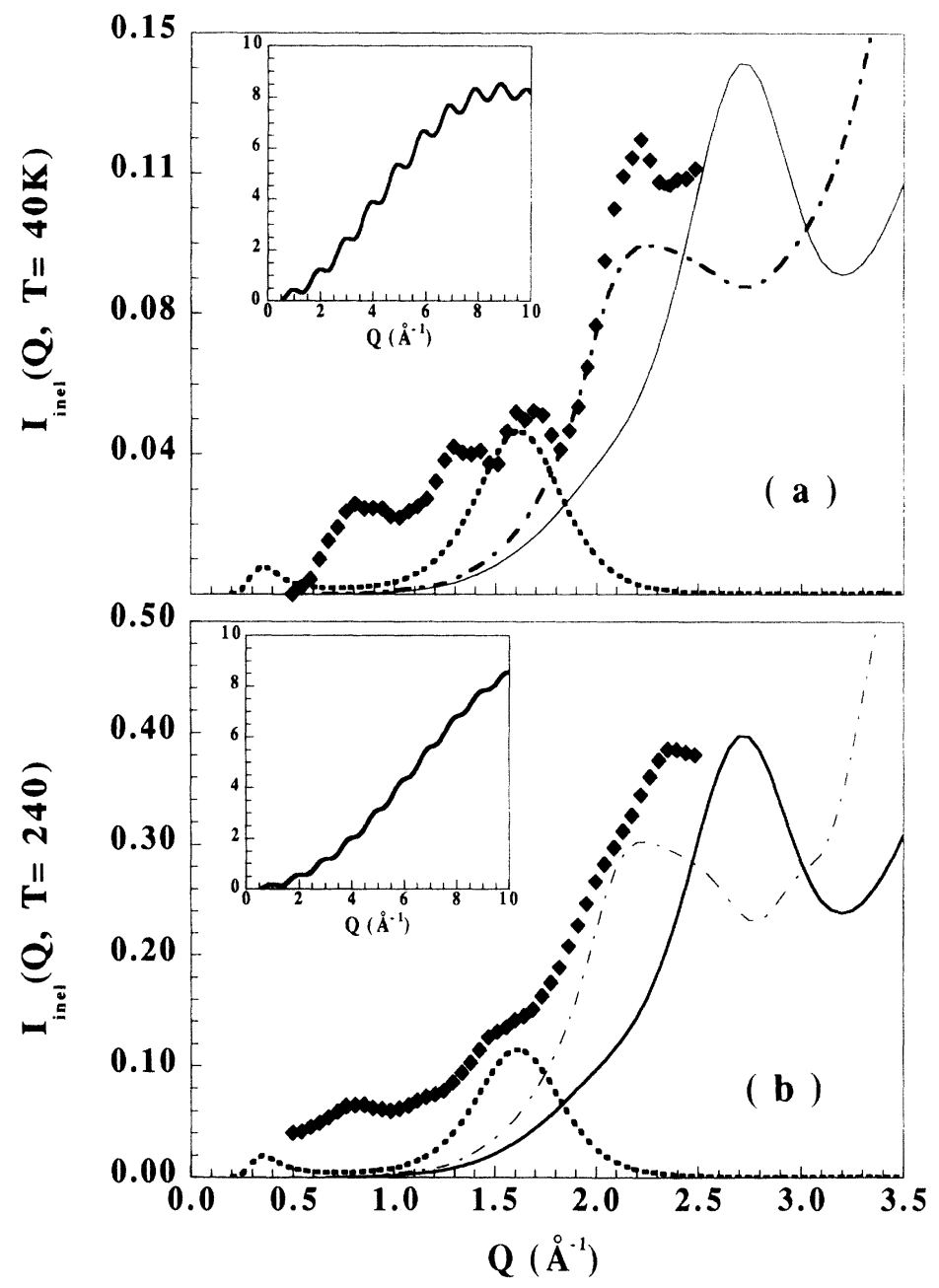

FIG. 4. A comparison of measured and modeled inelastic intensities for two representative temperatures. a) Low-temperature $(T=40 \mathrm{~K})$ data (one-phonon response). The experimental data are shown as filled lozenges, $(a)$ the solid line represents the inelastic intensity arising from excitations characterized by the average $\omega_{0}(Q)$ frequencies (see text), whereas the dash-dotted line (b) depicts those associated to $\omega_{l}(Q)$. The dotted line (c) shows the inelastic structure factor arising from excitations of a Se chain (bond torsions stretching and bendings). The inset shows the $I_{\text {inel }}^{\text {tors }}(Q)$ inelastic intensity arising from torsional oscillations as calculated Eq. (17) (see text). (b) Hightemperature $(T=240 \mathrm{~K})$ data (see text). Same symbols than above are used. The calculated curves correspond to a weighted sum of multiexcitation contributions (up to three phonon response) and the inset shows the response from torsional oscillations calculated including the two-phonon term as given in Ref. 11 . 
from the other chain motions (bond bending and stretchings) the inelastic structure factor corresponding to these motions can be calculated from ${ }^{11}$

$$
\begin{aligned}
I_{l, m}^{\mathrm{tors}}(Q) \propto & \frac{Q^{2}}{M_{\mathrm{Se}}}\left\{\frac{1}{3}\left(\gamma_{l} \cdot \gamma_{m}^{*}\right) j_{0}\left(Q r_{l m}\right)\right. \\
& \left.+\left[\frac{1}{3}\left(\gamma_{l} \cdot \gamma_{m}^{*}\right)-\frac{1}{r_{l m}^{2}}\left(\mathbf{r}_{l m} \cdot \gamma_{m}^{*}\right)\right] j_{2}\left(Q r_{l m}\right)\right\}
\end{aligned}
$$

where atoms $l, m$ are assumed to execute a torsional libration about a bond connecting two atoms to which $l, m$ are bonded. Since only atoms $l, m$ move, it is trivial to calculate the modulus of $\gamma_{l}=\gamma_{m}=1 / 2 M_{\mathrm{Se}}=6.3 \times 10^{-3}$ inverse atomic mass units, and the direction of the displacement vectors is tangential to the circle described by the motions of $l, m$ about the central bond. Because at thermal equilibrium the dihedral angle subtended by the four atoms is close to $\pi / 2$ (and therefore the angle between $\gamma_{l}, \gamma_{m}$ will be the same), ${ }^{22}$ the $j_{0}$ ( ) term in the above expression contribute very little to the intensity, being thus the pattern dominated by the $j_{2}($ ) term. The calculated pattern is shown in the inset of Fig. 4(a), where it can be seen that such motion gives rise to a rapid modulation of the inelastic intensity. Although such a model is an obvious oversimplification, since the correlated and simultaneous motion of several chain segments must be taken into account explicitly, it serves to assign an origin of the rapid oscillations.

The relatively low frequency of the torsional motion $(1.5 \mathrm{THz} \approx 50 \mathrm{~K})$ implies that multiexcitation contributions will become increasingly important even for moderately high temperatures (above $70 \mathrm{~K}$ ). To account for such effects, a simplified approach based upon the convolution approximation was followed, which employed the vibrational density of states derived from MD simulations at $T=15 \mathrm{~K}$ as a one-phonon distribution, and those regarding higher-order processes were calculated from Fourier transforms of products of such correlations. As shown in Fig. 4(b), multiphonon effects start to be considerable at temperatures about $100 \mathrm{~K}$ and above. However, as expected from the separation between dynamic and structural disorder effects given by Eq. (15), the net effect of including such terms is to alter the height of the calculated $I_{\text {inel }}(Q)$ curves without affecting the phase of the oscillations strongly. An explicit calculation of the two-phonon term corresponding to the simple torsional model can be easily made using formulas (22)-(28) of Ref. 11, and the calculated result is shown in the inset of Fig. 4(b). From comparison with the inset of Fig. $4(a)$, it can be seen that the progressive smearing out
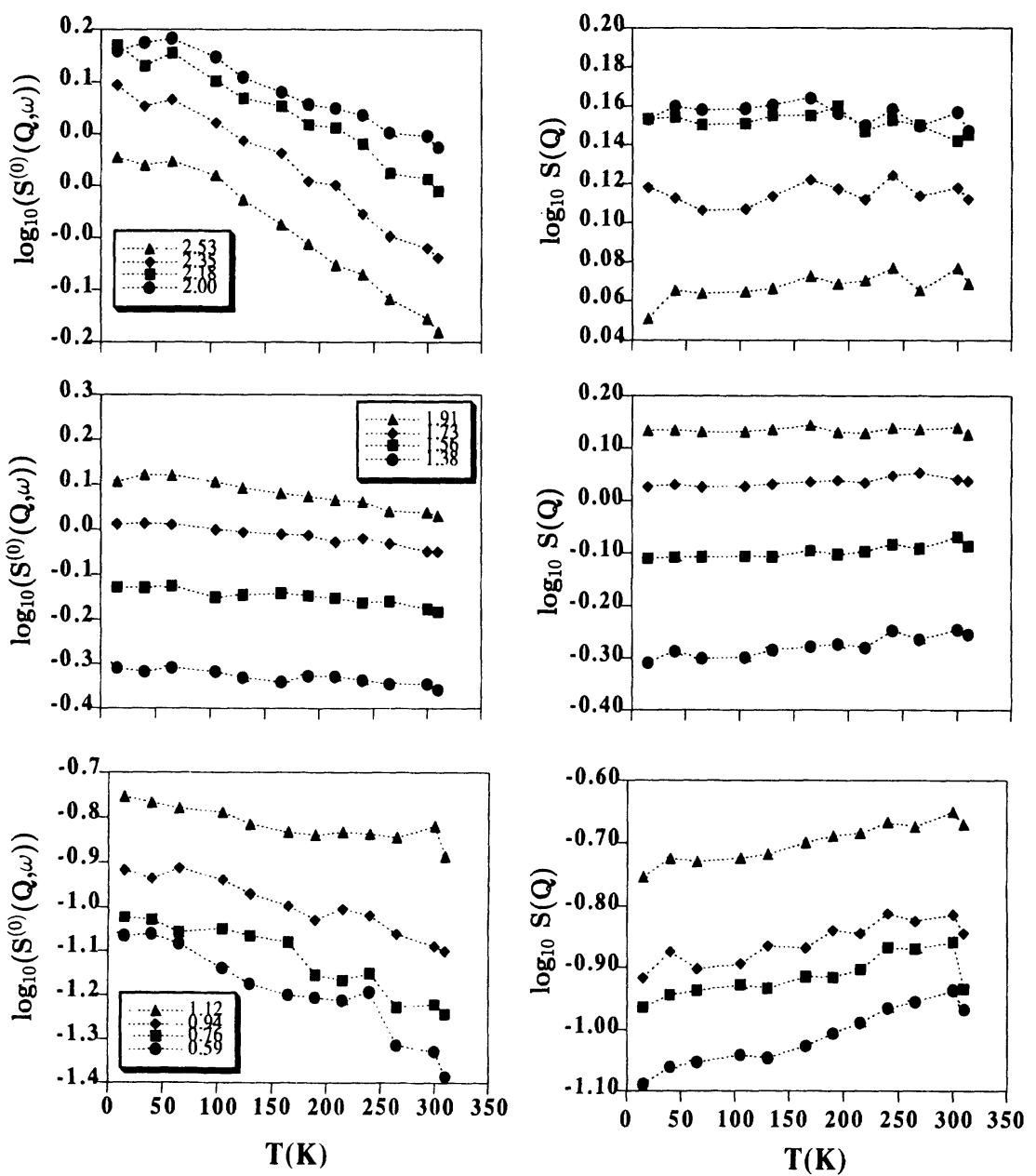

FIG. 5. Temperature dependence of the logarithm of both structure factors for a set of $Q$ values (given in the insets in $\AA^{-1}$ units) corresponding to regions well below $Q_{p} / 2\left(0.59\right.$ and $\left.0.76 \AA^{-1}\right)$, about $Q_{p} / 2 \quad(0.94$ and 1.12 $\left.\AA^{-1}\right),\left(Q_{p} 1.73-2.18 \AA^{-1}\right)$, and well above it. The same set of momentum- transfers for both sructure factors is used. 
of the higher-frequency oscillations of the experimental $I_{\text {inel }}(Q)$ curves can be explained in terms of multiexcitations of the lowest frequency localized librations, rather than in terms of the emergence of any new kind of excitation.

A set of graphs depicting the temperature dependence of $S^{(0)}(Q, \omega)$ and $S(Q)$ for several values of the momentum transfer is shown in Fig. 5. As can be seen, the dependence of $S^{(0)}(Q, \omega)$ with the temperature can be approximately described as a semilogarithmic decay with a slope strongly dependent on the wave vector. The point at $T=310 \mathrm{~K}$ experiences an additional strong falloff due to the proximity of the glass-transition temperature. ${ }^{23}$ On the other hand, the curves corresponding to $S(Q)$ evidence a more complex behavior with several different regimes clearly apparent. Assuming a logarithmic dependence with temperature, we have calculated from the fits the extrapolated values of both structure factors at $T=0$ $\mathrm{K}$ as well as the wave-vector dependence of the slopes, which are shown in Fig. 6. According with the result given by Eq. (11), the intensity of $S^{(0)}(Q, \omega)$ always de- cays with increasing temperature, however, the fact that the $Q$ dependence of such a decay has a minimum at about $Q \approx 1.5 \AA^{-1}$ (i.e., corresponding to a shoulder in the diffraction pattern) was by no means expected. In opposition, the temperature dependence of $S(Q)$ manifests a better known behavior, with maximum variation with temperature at low wave vectors, and a minimum is located close to the position of the peak in the diffraction pattern.

The structure factors extrapolated to $T=0 \mathrm{~K}$ are shown in Fig. 6(b). A comparison with that for the polycrystalline trigonal solid, also shown in the figure, exemplifies the amount of static disorder present in the glassy phase. The shoulder at about $1.6 \AA^{-1}$ and the main peak at $\approx 2 \AA^{-1}$ have a clear polycrystalline correlate, whereas the extra intensity appearing below $1 \AA^{-1}$ appears as characteristic of the glass phase.

From the value of the Debye temperature given by Meissner and Wobig, $\theta_{D}=130 \mathrm{~K},{ }^{24}$ the Debye-Waller factor corresponding to $S^{(0)}(Q, \omega)$ was evaluated from $W_{0}(Q)=\left(3 \hbar^{2} Q^{2} / 8 M_{\mathrm{Se}}\right)\left(1 / k_{B} \theta_{D}\right)$ giving $W_{0}(Q)=$
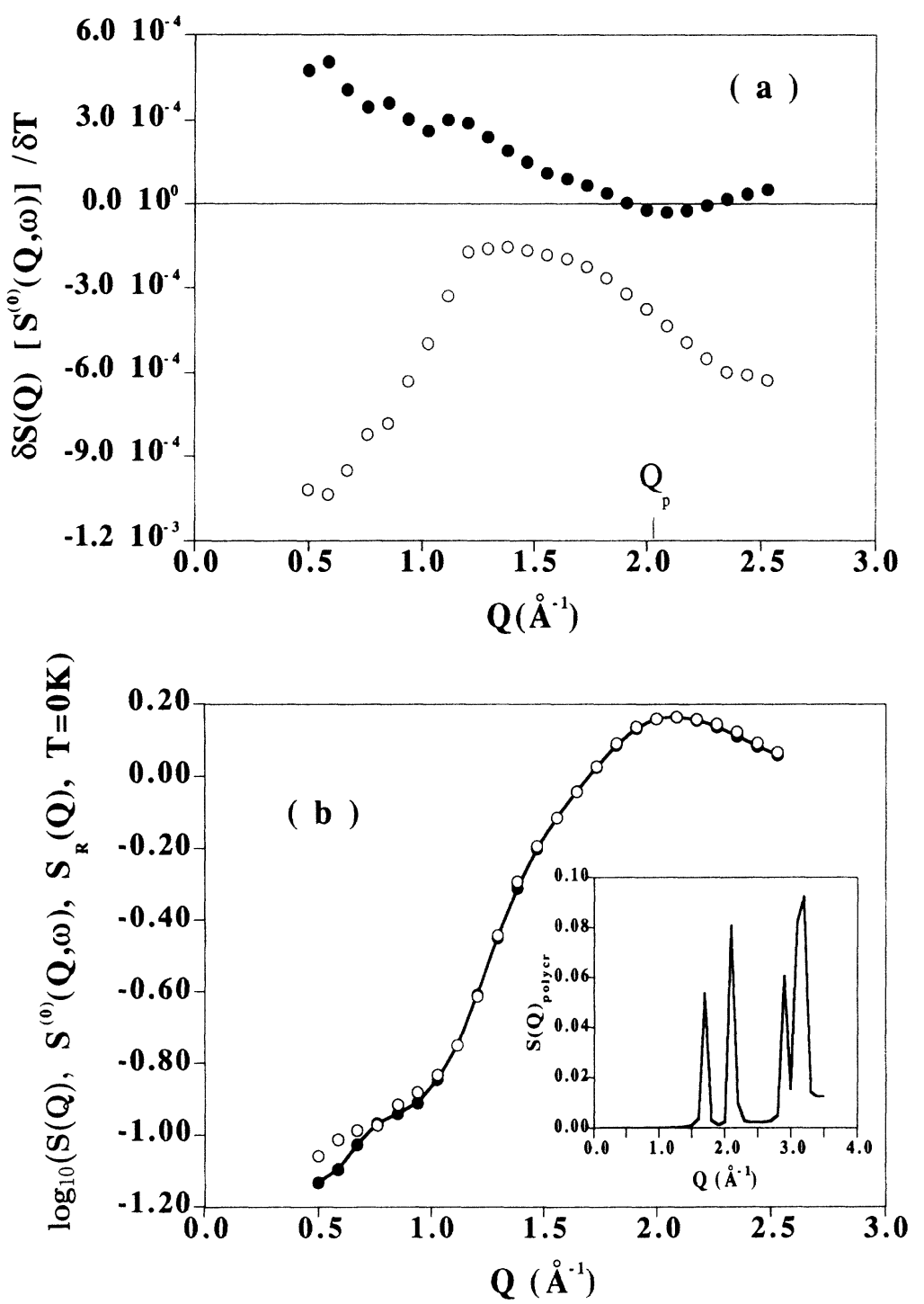

FIG. 6. (a) Wave-vector dependence of the change with temperature of the inelastic intensities. Open symbols represent quantities for $S^{(0)}(Q, \omega)$, and filled symbols for $S(Q)$. (b) Structure factors extrapolated to zero temperature. The inset shows the calculated structure factor for the polycrystalline (harmonic) trigonal solid. 
$Q^{2} 2.67 \times 10^{-4}$, and the extrapolated structure factors were then corrected from this zero-point motion effect. The resulting function $S_{R}(Q)$ is basically identical to $S(Q)$ within the restricted $Q$ range that we are exploring, and the most remarkable fact concerning this function regards the noticeable hump appearing at $Q \approx 0.76 \AA^{-1}$, which for this static quantity, could be interpreted as a signature of existence of intermediate range order with a characteristic length of $\approx 8.3 \AA$ (i.e., involving 7-8 Se atoms). It is also worth mentioning that such an intermediate length scale has been inferred in MD studies on the dynamics within the melt, ${ }^{25}$ as well as in some estimations regarding the thermal transport properties in the glass phase. ${ }^{26,27}$ The relevance of such a finding stems from the fact that it may constitute an indication of a scale renormalization so that most of the thermal and transport properties of the system are describable in terms of a coarse graining up to this length.

Finally, it is worth considering the estimates of the averaged mean-squared displacements calculated from the slopes of the $\ln \left[S^{(0)}(Q, \omega)-S(Q)+1\right]$ functions shown in Fig. 2. The present data were found to be in good agreement with that reported from the analysis of the quasielastic spectra, ${ }^{28}$ which were derived by means of averaging the spectra over a restricted range of wave vectors in order to suppress the strong coherent effects. However, the interesting point here is to compare such values with those obtained if the $Z(\omega)$ derived from simulational means is used to calculate the atomic displacements in the isotropic approximation. Once such an exercise is done, the resulting displacements are consistently higher than the experimental estimates, although the calculated and experimental results can be reconciled by assuming an effective mass for a Se atom larger than the free atom value. As a matter of fact, a value of $M_{\text {eff }}=2.4 M_{\text {Se }}$ makes the calculation closer to the experiment, a fact which may be interpreted as a consequence of the persistence of correlation effects. Experiments carried out averaging the spectra over a larger $Q$-range should be able to measure these quantities within the true single-particle regime, although the degradation in instrumental resolution for energy and momentum transfers will hamper such an endeavour.

In summary, the present study has evidenced the highly correlated nature of the atomic motions in glassy selenium by means of the analysis of experimental quantities, which can be accessed with relative ease. Such correlations are shown to give rise to noticeable structure in the wave-vector dependence of the structure factors, specially noticeable at low temperatures. Additional support for the assignment of the rapid oscillations in the inelastic intensities at moderate and low temperatures to localized (torsional) vibrations comes from the role played by these excitations regarding heat transport, as shown in a recent calculation. ${ }^{27}$

From the data presented in this paper it can be inferred that analogous phenomena should be apparent at least in covalent glasses, and, as a matter of fact, the picture that emerges regarding the motions as strongly collective in nature, involving up to 5-7 structural units (a single Se atom in this case) has a well established correlate in the well documented case of vitreous silica, ${ }^{4}$ where the collective reorientation of several $\mathrm{SiO}_{4}$ tetrahedra is assumed to be responsible for the observed thermal anomalies. Such findings seem to fit within the scenario sketched by Leggett and $\mathrm{Yu},{ }^{29}$ where the highly collective nature of the glassy dynamics is emphasized, although the lack, up to the authors knowledge, of a developed formalism, precludes any more detailed comparison.

\section{ACKNOWLEDGMENTS}

Work supported in part by Grant No. PB92-0114-C03 (DGICYT, Spain).
${ }^{1} \mathrm{~K}$. Suzuki, in Methods in Experimental Physics, edited by K. Sköld and D.L. Price (Academic, New York, 1987), Vol. 23, Chap. 7.

${ }^{2}$ See, for instance, F. Yonezawa, in Solid State Physics, edited by H. Ehrenreich and D. Turnbull (Academic, San Diego, 1991), Vol. 45, p. 179.

${ }^{3}$ See, for instance, J. Hafner, Phys. Rev. B 21, 406 (1980); and J.B. Suck, H. Rudin, H.J. Günterodt, and H. Beck, J. Phys. C 14, 2305 (1981), and references therein.

${ }^{4}$ U. Buchenau, M. Prager, N. Nucker, A.J. Dianoux, N. Ahmad, and W. A. Phillips, Phys. Rev. B 34, 5665 (1986).

${ }^{5}$ D.L. Price, Physica B 136, 25 (1986).

${ }^{6} \mathrm{~A}$ brief review is contained in S.R. Elliot, Physics of Amorphous Materials (Longman Scientific, Harlow, U.K., 1990), p. 185. An example of the comparison between experiment and computer simulation results for amorphous silicon can be found in W.A. Khamitakahara, C.M. Soukoulis, H.R. Shanks, U. Buchenau, and G.R. Grest, Phys. Rev. B 36, 6539 (1987); regarding Raman data for amorphous $\mathrm{Si}$ and Ge, see N. Maley, D. Beeman, and J.S. Lannin, Phys. Rev. B 38, 10611 (1988).
${ }^{7}$ Previous neutron and computer simulation results are described in M. Garcia-Hernandez, F.J. Bermejo, B. Fåk, J.L. Martinez, E. Enciso, N.G. Almarza, and A. Criado, Phys. Lett. A 175, 217 (1993); also M. Garcia-Hernandez, F.J. Bermejo, J. Alonso, A. Criado, F.J. Mompean, and J.L. Martinez, Phys. Rev. B 48, 149 (1993). See also references therein concerning previous work.

${ }^{8}$ Program SLABS, J. Alonso et al. Institut Laue Langevin technical Report No. 91AL01T, 1991.

${ }^{9}$ Program RESCAL, Risoe National Laboratory software package.

${ }^{10}$ J.M. Carpenter and D.L. Price, Phys. Rev. Lett. 54, 441 (1985); also U. Walter, D.L. Price, S. Susman, and K.J. Volin, Phys. Rev. B 37, 4232 (1988).

${ }^{11}$ J.M. Carpenter and C.A. Pelizzari, Phys. Rev. B 12, 2391 (1975); 12, 2397 (1975). The calculation of $I^{\text {tors }}(Q)$ follows the same steps than those given in J.M. Carpenter, J. Chem. Phys. 46, 465 (1969).

${ }^{12}$ J. Jäckle and K. Froböse, J. Phys. F 9, 967 (1979).

${ }^{13}$ Although the usual practice has been to replace such an average by the result for a cubic Bravais harmonic crystal 
$\left(\frac{1}{3} Q^{2} u^{2}\right)$ given in terms of a mean-square displacement $u^{2}$, it is worth recalling that the macroscopic isotropy condition of the glass should not be applicable at the length scales we are interested in, specially in cases where the interatomic bonding occurs along preferential spatial directions, see J. Krumhansl and H. Brooks, J. Chem. Phys. 21, 1663 (1953); H. Jobic, R.E. Gosh, and A. Renouprez, J. Chem. Phys. 75, 4025 (1981); and A. Griffin and H. Jobic, ibid. 75, 5940 (1981).

${ }^{14}$ S.R. Nagel, Phys. Rev. B 16, 1694 (1977).

${ }^{15}$ P.A. Reynolds, J.K. Kjems, and J.W. White, J. Chem. Phys. 56, 2928 (1972).

${ }^{16}$ F.J. Bermejo, M. Garcia-Hernandez, B. Fåk, J. Alonso, J.L. Martinez, and T. Mason, Europhys. Lett. 24, 545 (1993).

${ }^{17}$ W. Gotze, in Liquides, Crystallisation et Transition Vitreuse, Proceedings of the Les Houches Summer School of Theoretical Physics, City, 1989, edited by J.P. Hansen, D. Levesque, and J. Zinn-Justin (North-Holland, Amsterdam, 1989), p. 287; U. Bengtzelius, Phys. Rev. A 34, 5059 (1986); F. Mezei, W. Knaak, and B. Farago, Phys. Scr. T 19, 363 (1987).

${ }^{18}$ S. Susman, K.J. Volin, D.G. Montague, and D.L. Price, Phys. Rev. B 43, 11076 (1991).

${ }^{19}$ S.R. Elliot, J. Phys. Condens. Matter 4, 7661 (1992).

${ }^{20}$ R. Grosse, P. Krause, M. Meissner, and A. Tausend, J. Phys. C 11, 45 (1978).

${ }^{21}$ For plane-wave excitations in an isotropic continuum, the correlation function becomes identical to that given by Carpenter and Pelizzari (Ref. 11). For the single-particle limit (Einstein oscillators) explicit analytical expressions are given in Ref. 12 as well as an interpolation scheme defined by a coherence length chosen to fit the data. Note that in the present application of such a model the relevant wave vectors are calculable from the "dispersion relations" and associated polarizations given in a previous report, where it may be seen that the average $\omega_{0}(Q)$ mostly encompass sound wave and lower optic excitations, whereas $\omega_{l}(Q)$ contains contributions arising from higher optical modes.

${ }^{22}$ N.G. Almarza, E. Enciso, and F.J. Bermejo, Europhys. Lett. 17, 595 (1992).

${ }^{23} \mathrm{~A}$ more detailed study on the inelastic intensities about the glass transition is given by G. Carini, M. Cutroni, G. Galli, and F. Wanderlingh, J. Non-Cryst. Solids 30, 61 (1978).

${ }^{24} \mathrm{M}$. Meissner and D. Wobig, in The Physics of Selenium and Tellurium, Springer Series in Solid State Sciences, Vol. 13, edited by E. Gerlach and P. Grosse (Springer-Verlag, Berlin, 1979), p. 68.

${ }^{25}$ N.G. Almarza, E. Enciso, and F.J. Bermejo, J. Chem. Phys. 99, 6798 (1993).

${ }^{26}$ F.J. Bermejo, in Proceedings of the II International Discussion Meeting on Relaxations in Complex Systems, Alicante, Spain, 1993 [J. Non. Cryst. Solids (in press)].

${ }^{27}$ F.J. Bermejo, M. Garcia-Hernandez, J.L. Martinez, and A. Criado, Phys. Rev. B 49, 8689 (1994).

${ }^{28} \mathrm{R}$. Zorn and U. Buchenau, Europhys. Lett. 18, 523 (1992).

${ }^{29}$ A.J. Leggett, Physica B 169, 322 (1991); see also C.C. Yu and A. J. Leggett, Comments Cond. Mater Phys. 14, 231 (1988). 